\title{
Influence of pilates method on the treatment of nonspecific chronic low back pain: a protocol study for a randomized, controlled, blind trial
}

\author{
Bianca Martins de Souza', Daiane Oliveira da Silva', Lais Paraguai Justo', Leandro Lazzareschi', \\ Daniela Aparecida Biasotto-Gonzalez², Igor Phillip dos Santos Glória'
}

\begin{abstract}
Background: Low back pain is one of the most common musculoskeletal problems among workers. Studies estimate that $90 \%$ of the adults will suffer at least one episode of low back pain in life that will lead to the temporary interruption of work. A type of exercise that has gained increasing popularity among individuals with low back pain in the last decade is the Pilates method. Objective: Determine the influence of the Pilates method on improvements in pain, kinesiophobia, central sensitization and function in patients with chronic nonspecific low back pain. Methods: Thirty-four male and female volunteers between 18 and 35 years of age with a complaint of nonspecific low back pain for at least three months will be divided into two groups: Pilates method and control group. An evaluation chart created by the authors will be used to collect data on personal information, history of diseases, alcohol use, smoking, pain intensity (visual analog scale) and medications used. The Tampa Scale for Kinesiophobia, Central Sensitization Questionnaire and Oswestry Disability Index will also be administered. Pilates exercises will be performed three time per week for four weeks and administered by a researcher with adequate training in the method. Reevaluations will be performed after the sixth treatment session in the Pilates group, two weeks after the initial evaluation in the control group, after the $12^{\text {th }}$ session in the Pilates group and after four weeks in the control group. Another reevaluation will be performed three months after treatment. Results: The data will be submitted to statistical analysis and the level of significance will be set to $5 \%$. Conclusion: Based on the results analyzed it will be possible to determine the influence of Pilates Method on improvements in pain, kinesiophobia, central sensitization and function in patients with chronic nonspecific low back pain.

Key words: low back pain, Pilates, kinesiophobia, central sensitization.
\end{abstract}

\section{BACKGROUND}

Nonspecific low back pain is defined as pain and discomfort located between the lower edge of the ribs and lower gluteal folds (L1 to L5) that is not associated with a specific pathological condition and is characterized as chronic when lasting 12 or more weeks ${ }^{(1)}$. Chronic low back pain may be associated with central sensitization, which is a proposed physiological phenomenon in which dysregulation of the central nervous system causes neuronal dysregulation and hyperexcitability, resulting in hypersensitivity to both nocive and non-novice stimuli( ${ }^{(2)}$. Central sensitization is not predominated by local inflammation or neural injury, but is rather a prolonged state of hyperexcitability of the neural pathways of pain and pain-processing areas in the brain and is therefore associated with chronic pain ${ }^{(3)}$.

Kinesiophobia is another relevant point related to the worsening of a pain condition. As the name suggests, kinesiophobia is the fear of movement. The anticipation of pain due to movement makes affected individuals gradually diminish their activities, which limits their functions and restricts participation in activities of daily living ${ }^{(4)}$.

We live in a world of mechanized, automated activities and the need to increase production at work, which exposes workers to extreme pressure that can lead to repetitive strain injury and work-related musculoskeletal disorders. When not treated adequately, these conditions and more complex syndromes contribute to a longer of time on leave from work and disability, leading to increases in both public and private healthcare costs ${ }^{(5,6)}$.

Low back pain is one of the most common musculoskeletal problems among workers. Risk factors for this condition include repetitive twisting movements of the spine, pushing or pulling objects, trips, falls and poor posture ${ }^{(7)}$. Studies estimate that $90 \%$ of the adults will suffer at least one episode of low

This study is registered with Clinical Trials under protocol number: NCT03854643.

Correspondence: Igor Phillip dos Santos Glória. Department of Physical Therapy, Universidade de Mogi das Cruzes (UMC), Av. Dr. Cândido Xavier de Almeida Souza, 200 Centro Cívico, Mogi das Cruzes - SP, 08780-911, Brazil. Phone: + 5511 99546-6201. E-mail: igorgloria@yahoo.com.br

Department of Physical Therapy, Universidade de Mogi das Cruzes (UMC), Mogi das Cruzes (SP), Brazil.

Full list of author information is available at the end of the article.

Financial Support: nothing to declare.

Submission date 09 December 2019; Acceptance date 27 April 2020; Publication date 27 May 2020 
back pain in life that will lead to the temporary interruption of work ${ }^{(8)}$.

The $21^{\text {st }}$ century has been marked by the search for quality of life and wellbeing ${ }^{(9,10,11)}$. Individuals are more aware of the importance of avoiding a sedentary lifestyle and practicing physical exercise. Indeed, the scientific community has published innumerous articles on the importance of regular physical activity as a way of preventing and even curing diseases, such as cancer and depression ${ }^{(12,13)}$.

A type of exercise that has gained increasing popularity among individuals with low back pain in the last decade is the Pilates method, which was developed in the 1920 s by Joseph Pilates and consists of whole-body conditioning with the aim of developing better body awareness and improving posture ${ }^{(13)}$.

Currently, no other method has grown and spread as rapidly as Pilates. In large urban centers, nearly all athletic academies and muscle-building gyms have at least one Pilates studio $^{(14)}$.

Since its creation, the method has been based on six principles, known as the classic principles: breathing, centering, control, precision, flow and concentration. Pilates exercises mainly involve isometric contractions of core muscles responsible for the stabilization of the spinal column during both movement and at rest. The benefits include improvements in strength, range of motion, coordination, balance, muscle symmetry, flexibility and proprioception ${ }^{(15)}$.

Despite its popularity, the efficacy of the pilates method on chronic low back pain is debated in the literature and a systematic review was published demonstrated positive effects of the Pilates method in the treatment of chronic low back pain ${ }^{(16)}$. Therefore, the aim of the proposed study is to determine the effect of this method on chronic nonspecific low back pain.

\section{JUSTIFICATION}

Low back pain is a considerable health problem throughout the world, with an evident socioeconomic impact. Chronic low back pain affects $23 \%$ of the population at some point in life. As health resources are scarce, the cost-benefit relationship of treatment for low back pain is considered as important as its effectiveness. Clinical practice guidelines recommend exercise for patients with low back pain, which exerts an influence on the endogenous inhibitory system, inducing hypoalgesia. Moreover, kinesiophobia seems to be related to pain and disability in patients with low back pain and exercise can offer benefits to improve these psychological factors.

\section{HYPOTHESIS}

The hypothesis of this study is that core stabilization using the Pilates method is capable of promoting improvements in pain, kinesiophobia, central sensitization and function in patients with chronic nonspecific low back pain.

\section{METHODS}

\section{Overview of research design}

A randomized, controlled, blind, clinical trial will be conducted to investigate the influence of core stabilization using the Pilates method on pain, kinesiophobia, central sensitization and function in patients with chronic nonspecific low back pain.

\section{Approval and registry}

This project received approval from the Human Research Ethics Committee of University of Mogi das Cruzes (certificate number: 90158318.3.0000.5497) and is registered with clinicaltrials.gov (NCT 03854643).

The study will be developed at the polyclinic of Universidade de Mogi das Cruzes, located the following address: Rua Dom Antônio Cândido de Alvarenga, 170 - Centro, Mogi das Cruzes - SP, CEP 08780-070. The study will involve four evaluations: pre-intervention, after two weeks of intervention, after four weeks of intervention and three months after the end of the intervention.

Study hypothesis: Core stabilization using the Pilates method is capable of promoting improvements in pain, kinesiophobia, central sensitization and function in patients with chronic nonspecific low back pain.

Null hypothesis: Core stabilization using the Pilates method is not capable of promoting improvements in pain, kinesiophobia, central sensitization and function in patients with chronic nonspecific low back pain.

\section{Volunteers}

Thirty-four male and female volunteers between 18 and 35 years of age with a complaint of nonspecific low back pain for at least three months will be divided into two groups: 17 submitted to the Pilates method and 17 in the control group.

\section{Inclusion criteria}

Low back pain grade 3 or higher for 12 or more weeks not related to any pathology or specific cause. The volunteers cannot be undergoing any physiotherapeutic or medicinal treatment.

\section{Exclusion criteria}

Low back pain lower than grade 3 and/or related to a disease or specific cause, herniated disc, a history of spinal fractures, tumors, pregnancy and signs of neurological compression. Volunteers undergoing physiotherapeutic and/or medicinal treatment will also be excluded.

\section{MATERIALS}

An evaluation chart created by the authors will be used to collect data on personal information, history of diseases, alcohol use, smoking, medications used and pain intensity. 
Pain intensity will be rated using the visual analog scale(8), which ranges from 0 (absence of pain) to 10 (unbearable pain). The Tampa Scale ${ }^{(17)}$ for Kinesiophobia will also be used, which is a 17-item scale on which the volunteer shows agreement or disagreement to the statement posed. Each item is scored from 1 to 4 points.

The Central Sensitization Questionnaire(18) will be employed. This questionnaire is divided into two parts. Part A has 25 questions addressing the occurrence of pain in the previous three months, with responses ranging from "never" (score 0 points) to "always (scored 4 points). Part $B$ has 10 questions addressing possible medical diagnoses, with a "yes/no" response for each one.

The Oswestry Disability Index 2.0 ${ }^{(19)}$ will also be used. This index has ten sections, each with six statements. The volunteer marks the statement that best fits his/her current condition.

\section{PROCEDURES}

Following approval from the ethics committee of the university, thirty-four volunteers will be recruited based on the eligibility criteria. All volunteers who agree to participate will sign a statement of informed consent. The participants will then be evaluated by Researcher A. All evaluations and the intervention will be conducted at the polyclinic of Universidade de Mogi das Cruzes.

\section{Evaluation}

A blinded researcher will be in charge of the evaluation of the volunteers (Figure 1). The personal data will first be collected using the evaluation chart created by the researchers. Next, the Tampa Scale for Kinesiophobia, Central Sensitization Questionnaire and Oswestry Disability Scale will be administered (in that order). The researcher will not be aware of the allocation of the volunteers to the different groups.

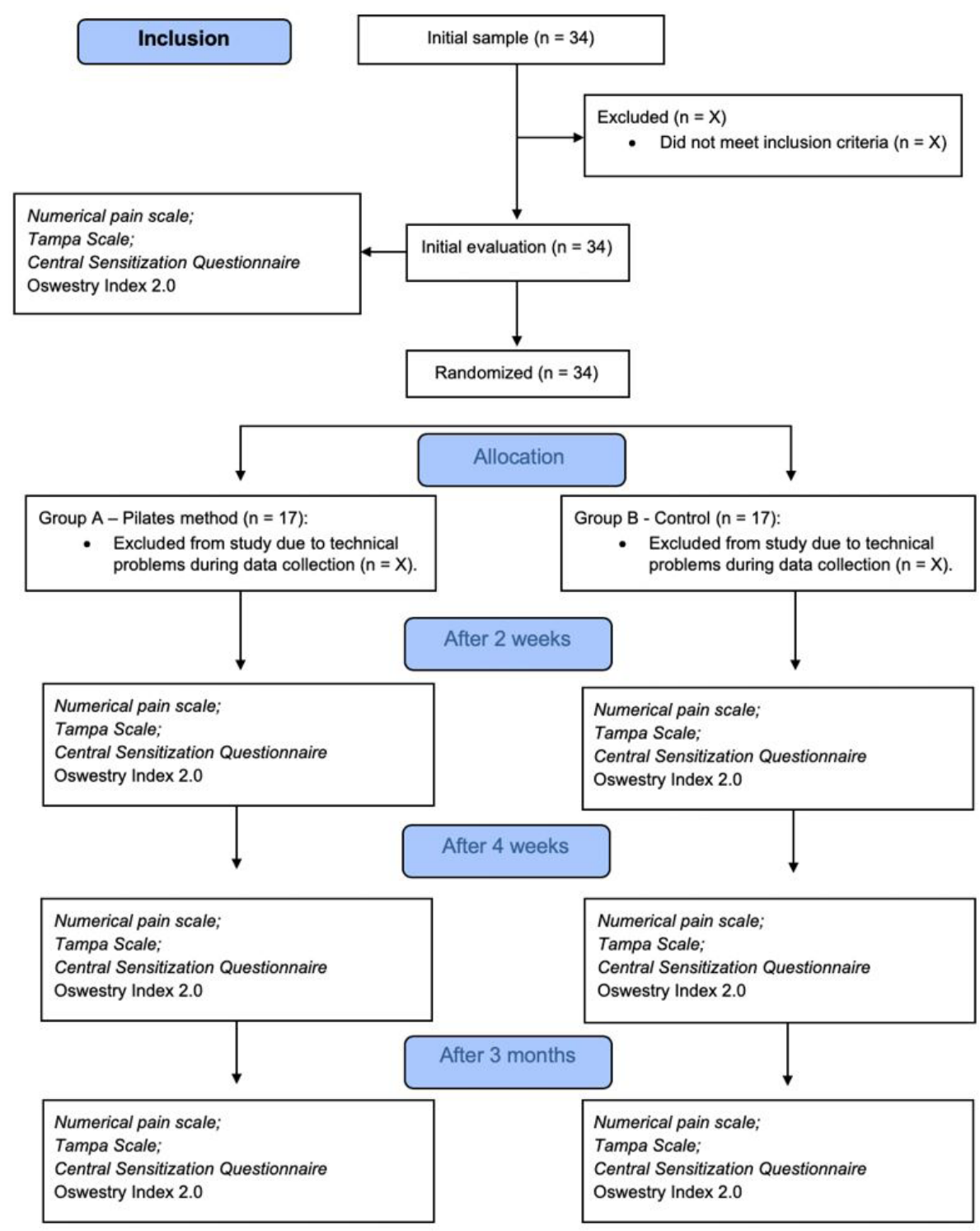

Figure 1: Flowchart 


\section{Randomization and blinding}

After the initial evaluation by Researcher A, Researcher B will perform the randomization procedure to allocate the volunteers to the different groups (Pilates and control) using block randomization with the aid of the randomization. com site. Seventeen individuals will be allocated to each group. Researcher $\mathrm{C}$ will be in charge of the treatment of the volunteers and will not have access to the evaluation charts. After treatment and the reevaluations, Researcher $D$ will be in charge of the statistical analysis of the data and will be blinded to the allocation of the volunteers to the different groups.

\section{Reevaluations}

Reevaluations will be performed after the sixth treatment session in the Pilates group, two weeks after the initial evaluation in the control group, after the $12^{\text {th }}$ session in the Pilates group and after four weeks in the control group. Another reevaluation will be performed three months after treatment to analyze the effectiveness of Pilates method at maintaining the improvement in the clinical state of the patients. All reevaluations will be performed using the sample parameters and scales employed during the pre-intervention evaluation by the same researcher, who will be blinded to the allocation of the volunteers to the different groups. The volunteers allocated to the control group will be offered treatment with the Pilates method after the completion of the data collection.

\section{Treatment program with Pilates method}

The Pilates exercises will be performed three times per week for four weeks (total: 12 sessions) at the policlinic of Universidade de Mogi das Cruzes. Each session will last 40 minutes and will be administered by a researcher with previous training in the method.

In the first session, the volunteers will be trained individually to activate the "powerhouse", which is isometric contraction of the transverse abdominal muscle, perineum, gluteal muscles and multifidus muscles during diaphragmatic breathing. In the next session, the participants will begin specific training while remembering the activation. Ten exercises will be performed per session in accordance with the limitations of each participant, with each exercise repeated no more than three times. All exercises will be adapted and modified, with three levels of difficulty: basic, intermediate and advanced. For example, "hip abductor" is a basic exercise in which the participant sits with the lower limbs in abduction and places one hand on the contralateral foot. The level of difficulty on this exercise is increased by increasing the range of adduction of the lower limbs with flexion of the trunk until touching the contralateral foot with both hands. Likewise, the "roll up" is an advanced exercise in which the participant is placed in the supine position, flexes the trunk and contracts the abdominal muscles until the ribs are no longer touching the mat, with the hands extended toward the feet. To make this exercise easier, the participants will be instructed only to perform isometric contraction of the abdomen, lifting the scapulas off the mat until the entire back is raised and touching the feet with the hands.

When adaptations are not possible, the exercise will be replaced with another one with a similar objective. The level of difficulty of each exercise will be defined according to the needs of each participant and increased as the participants learn to execute each exercise correctly without postural compensation or pain, such as an increase in the number of repetitions (no more than three) and the reach of each movement. The exercises will be performed individually by each participant. The complete description of the exercises used in treatment (from the initial to the final position, number of repetitions and photo of each exercise) will be presented in the protocol of the study.

\section{DISCUSSION}

The aim of this study is to determine the influence of the Pilates method (three sessions per week for four weeks) on improvements in pain, kinesiophobia, central sensitization and function in patients with chronic nonspecific low back pain. Chronic low back pain has been the target of studies involving the use of physical and therapeutic exercises. Thus, there is a need to investigate the effect of the Pilates method in the treatment of this condition.

\section{Acknowledgments:}

The authors are grateful for all the support given by Mogi das Cruzes University as well as all researchers and volunteers who participated in the study.

\section{AUTHOR'S CONTRIBUTIONS:}

All cited authors participated in these steps. BMS: conception and design, data collection and analysis, manuscript writing and final approval of the manuscript. DOS: conception and design, manuscript writing, final approval of manuscript. LPJ: critical revision and final approval of the manuscript. LL: critical revision and final approval of the manuscript. DABG: critical revision and final approval of the manuscript. IPSG: conception and design, financial support, manuscript writing, final approval of manuscript.

\section{CONFLICTS OF INTEREST:}

nothing to declare.

\section{AUTHORS DETAILS}

2 Postgraduate Program in Rehabilitation Sciences, Universidade Nove de Julho (UNINOVE). Água Branca, São Paulo (SP), Brazil. 


\section{REFERENCES}

1. Valenza MC, Rodriguez-Torres J, Cabrera-Martos I, Diaz-Pelegrina A, Aguilar-Ferrandiz ME, Castellote-Caballero Y. (2016). Results of a Pilates exercise program in patients with chronic non-specific low back pain: a randomized controlled Trial.

2. NEBLETT R, COHEN H, CHOI Y, HARTZELL MM, WILLIAMS M, MAYER TG, GATCHEL RJ. (2013). The central sensitization inventory (CSI): establishing clinicallysignificant values for identifying central sensitivity syndromes in na outpatient chronic pain sample. J pain; 14 (5): 438-445.

3. SMART KM, BLAKE C, STAINES UM, DOODY C. (2011). Discriminative validity of "nociceptive", "peripheral neuropathic" and "central sensitization" as mechanisms-based classifications of musculoskeletal pain. Clin J pain; 27:655-663.

4. CARAVIELLO EZ, WASSERSTEIN S, CHAMLIAN TR, MASIERO D. (2005). Avaliação da dor e função de pacientes com lombalgia tratados com um programa de Escola da Coluna. Acta Fisiátrica, v. 12, n. 1, p. 11-14.

5. MATTOS R, SAMANO T. (2005). Intervenção do Pilates na reeducação postural: um estudo de caso. Atelier do Corpo.

6. O'SULLIVAN PB. (2000). Lumbar Segmental instability: clinical presentation and specific stabilizing exercise management. Manual Therapy 5(1).

7. FONSECA JL, MAGINI M, de FREITAS TH. (2009). Laboratory gait analysis in patients with low back pain before and after a Pilates intervention. J Sport Rehabil; 18(2): 269-282.

8. SALTER RB. (2001). Distúrbios e lesões do sistema musculoesquelético. Medsi 3 ed.

9. ALVES A, CHUQUI E, SOUZA C, ROSSI L. (2009). Grau de satisfação com a imagem corporal em praticantes de Pilates. Revista digital - Buenos Aires - Ano 13, n. 128.

10. PIRES DC, DE SÁ CKC. (2005). Pilates: notas sobre aspectos históricos, princípios, técnicas e aplicações. Efdeportes.com 10(91):1-4.

11. SACCO LCN, ANDRADE MS, SOUZA PS, NISIYAMA M, CANTUARIA AL, MAEDA FYI, PIKEL M. (2005). Método Pilates em revista: aspectos biomecânicos de movimentos específicos para reestruturação postural - estudo de caso. Rev. Bras. Ciên. e Mov. 13(4): 65-78.

12. EYIGOR S, KARAPOLAT H, YESIL H, USLU R, DURMAZ B. (2010). Effects of Pilates exercises on functional capacity, flexibility, fatigue, depression and quality of life in female breast câncer patients: a randomized controlled study. Eur J Phys Rehabil Med 46(4): 481-487.

13. KEAYS KS, HARRIS SR, LUCYSHYN JM, MACLNTYRE DL. (2008). Effects of Pilates exercises on shoulder range of motion, pain, mood and upper-extremity function in women living with breast cancer: a pilot study.Phys Ther v. 88, ed. 4, p. 494-510.

14. ALLEN M. (2009). The Power of Pilates. Nursing \& Residential Care, v. 11, n. 10.

15. ROBLE JR. (2015). Uma interpretação estética do método Pilates: seus princípios e convergências com a Educação Somática. Rev. Bras. Estud. Presença vol. 5 no.1.

16. LIN HT, HUNG WC, HUNG JL, WU PS, LIAW LJ, CHANG JH (2016). Effects of Pilates on patients with chronic non-specific low back pain: a systematic review. J Phys Ther Sci. 28(10: 2961-2969.

17. TURK D, MELZACK R. (1992). Handbook of Pain Assessment. 2nd ed. New York, NY: The Guilford Press.

18. DE SOUZA FS, MARINHO CDA S, SIQUEIRA FB, et al. Psychometric testing confirms that the Brazilian-Portuguese adaptations, the original versions of the Fear-Avoidance Beliefs Questionnaire, and the Tampa Scale of Kinesiophobia have similar measurement properties. Spine (Phila Pa 1976). 2008;33:10281033.

19. MAYER TG, NEBLETT R, COHEN H. et al.(2012). The development and psychometric validation of the central sensitization inventory. Pain Pract, 12(4), 276-285.

20. VIGATTO R, ALEXANDRE NM, CORREA FILHO HR. Development of a Brazilian Portuguese version of the Oswestry Disability Index: cross-cultural adaptation, reliability, and validity. Spine (Phila Pa 1976). 2007;32(4):481-6

21. Schulz KF, Altman DG, Mohe D. CONSORT 2010 Statement: updated guidelines for reporting parallel group randomised trials CONSORT Statement. 